\title{
Experimental Acoustic Determination of the Void Fraction in Two-Phase Flow in Horizontal Pipelines
}

\author{
Fernando Augusto de Noronha Castro Pinto ${ }^{1}$, Thiago de Campos Negrelly ${ }^{1}$ and Alexandre Silva de Lima ${ }^{1,2}$ \\ 1. Mechanical Engineering Department, UFRJ (Universidade Federal do Rio de Janeiro), Rio de Janeiro 21941-972, Brasil \\ 2. Mechanical Engineering Department, CEFET/RJ (Centro Federal de Educação Tecnológica Celso Suckow da Fonseca), Rio de \\ Janeiro 20271-110, Brasil
}

Received: April 08, 2014 / Accepted: April 28, 2014 / Published: August 25, 2014.

\begin{abstract}
In multi-phase flows, the phases can flow and arranged in different spatial configurations in the pipe, which called flow patterns. This type of flow is found in the oil, chemical and nuclear industries. For example, in the production and transport of oil and gas, the identification of the flow patterns are essential for answering those questions which are related to the economic return of the field, such as, measuring the volumetric flow, determining the pressure drop along the flow lines, production management and supervision. In offshore production, these factors are very important. This paper presents a new method for measuring the void fraction in horizontal pipelines, taking the air as gas in water-air two-phase flow. Through acoustic analysis of the frequency response of the pipe, the method gets the parameters to changes in runoff regime, in an experimental arrangement constructed on a small scale. The main advantages are the non-intrusive characteristic and easy to implement. The paper is composed of a qualitative experimental evaluation and transducers (microphone) which are used to analyze variations in the response accompanying variations in void and flow pattern changes. Changes are imposed and controlled by a two-phase flow experimental simulation rig, including a measurement cell constituted of an external casing that can isolate the measurement from the environmental background noise fitted with acoustic pressure transducers radially arranged, and the impact of a monitored excitation mechanism. The signals which captured by the microphones are processed and analyzed by checking their frequency contents changes according to the amount of air in the mixture.
\end{abstract}

Key words: Experimental method, two-phase flow, determination of void fraction, horizontal pipelines.

\section{Introduction}

Multiphase flows are present in the oil, chemical and nuclear industries, among others. In this type of flow, the phases can be arranged in different configurations inside the pipeline, which called flow patterns. In the production and transport of oil and gas, identifying the flow patterns is essential to the economic return of the field, in the measurement of volumetric flow and in determining the pressure drop along the flow lines, production management and supervision [1]. These factors are critical in offshore production, due to the large distances and high costs. In the oil industry, the problems which associated with multiphase flow have

Corresponding author: Alexandre Silva de Lima, D.Sc., research fields: signal processing, robotic, machine design, hydraulic and pneumatic systems. E-mail: alima@cefet-rj.br. long been studied [2]. Thus, the knowledge of the void fraction in the flow is important for the design, operation and maintenance of oil production and transport facilities.

Section 2 presents the objectives; Section 3 shows the bi-phasic flow; Section 4 appears the experimental modeling; Sections 5 and 6 present the results and conclusions, respectively.

\section{Objectives}

The conventional assessment of the void fraction in offshore oil production is usually expensive, heavy and large. Moreover, they present a lag between the time when the bi-phasic mixture is occurring and the instant of measurement. Modern techniques can make the measurement in real time, but it is still expensive and 
usually intrusive. The aim of this study is to present an acoustic-based method to measure the void fraction in a flow. It will be tested in a rig with water-air flow in different conditions.

\section{Bi-phasic Flow}

The flow of oil, water, gas and sediments, are called multiphase flow. Nevertheless, in the current work, a two-phase flow, one phase gaseous and the other liquid will be considered. Besides the dimensioning of production pipelines based on pressure drop, it is important to determine the fluid composition in the pipeline in various flow conditions and allow the proper design of the separating system of the process plant [3].

\subsection{Gas Volume Fraction}

The gas volume fraction $\left(H_{g}\right)$ is defined as the ratio of the volume in a pipe segment, which occupied by the gas and the total volume of pipe segment in Eq. (1).

$$
H_{g}=\frac{V_{\text {gas }}}{V_{\text {pipe }}}
$$

The volume fraction of gas is a fraction ranging from 0 (liquid flow only) to 1 (gas flow only).

The remaining of the pipe segment is occupied by the liquid, called the volume fraction of the liquid $\left(H_{L}\right)$, Eq. (2).

$$
H_{L}=1-H_{g}
$$

\subsection{No-Slip Gas Volume Fraction}

The no-slip gas volume fraction $\left(\lambda_{g}\right)$ is defined as the ratio of the volume of gas in a pipe segment, divided by the volume of the pipe segment that would exist, if the gas and the liquid were flowing at the same speed (no-slip).

The calculation can be done using known flow rates of gas and liquid as shown in Eq. (3).

$$
\lambda_{g}=\frac{q_{g}}{\left(q_{g}+q_{L}\right)}
$$

where, $q_{L}$ is the liquid flow rate and $q_{g}$ is the gas flow, respectively.

The no-slip gas volume fraction $\left(\lambda_{L}\right)$ is defined in Eq. (4).

$$
\lambda_{L}=1-\lambda_{g}=\frac{q_{L}}{\left(q_{g}+q_{L}\right)}
$$

\subsection{Velocity}

Many correlations for two-phase flow are based on a variable, called superficial velocity [4]. The superficial velocity of a fluid phase is defined as the speed, this phase would be subjected if the flow would use the whole cross section of the tube.

\subsubsection{Superficial Velocity of the Gas}

The superficial velocity of gas velocity is calculated in Eq. (5).

$$
v_{s g}=\frac{q_{g}}{A}
$$

where, $A$ is the cross section of the tube.

The real velocity of the gas is calculated in Eq. (6).

$$
v_{g}=\frac{q_{g}}{A \cdot H_{g}}
$$

\subsubsection{Superficial Velocity of the Liquid}

The superficial velocity of the liquid is calculated in Eq. (7).

$$
v_{S L}=\frac{q_{L}}{A}
$$

The real velocity of the liquid is calculated in Eq. (8).

$$
v_{L}=\frac{q_{L}}{A \cdot H_{L}}
$$

\subsubsection{Superficial Velocity of the Bi-phasic Mixture}

The superficial velocity of the bi-phasic mixture is the sum of the superficial velocities of the phases (Eq. (9)).

$$
v_{m}=v_{L}+v_{g}
$$

\subsection{Non-slip Velocity}

The non-slip velocity is defined as the difference between the actual speeds of the gas and liquid phases (Eq. (10)). 


$$
v_{s}=v_{g}-v_{L}=\frac{v_{s g}}{H_{g}}-\frac{v_{s L}}{H_{L}}
$$

The no-slip volume fraction is calculated in Eq. (11).

$$
\lambda_{g}=\frac{v_{s g}}{v_{m}}
$$

\subsection{Determination of the Properties of the Liquid Mixture}

When the liquid mixture contain oil and water, a way to measure the effect of these phases is used, which the proportionality factor is defined in Eq. (12).

$$
\operatorname{prop}_{l}=\operatorname{prop}_{o}\left(\frac{q_{o}}{q_{o}+q_{a}}\right)+\operatorname{prop}_{a}\left(1-f_{o}\right)
$$

where, the index "o" refers to oil; the index " $a$ " refers to water; and the index "l" refers to the liquid mixture.

\subsection{Acoustics and Cylinder Vibration Mode}

When a container is filled with liquid, its natural frequency is modified. This is a property of the system and it is independent of the magnitude of the active force. Eq. (13) presents the equation of flexural vibrations in beams with free ends and without effects of load.

$$
E I \frac{\partial^{4} v}{\partial x^{4}}+m \frac{\partial^{2} v}{\partial t^{2}}=0
$$

where, $m$ is the total mass of the pipe plus the components of the bi-phasic mixture water and air, per unit of the length; and $v$ is the cross displacement of the uniform pipe with product of the EI stiffness.

The first natural frequency form is presented in Eq. (14).

$$
f=\beta^{2} \sqrt{\frac{E I}{\rho A}}
$$

where, $\rho$ is the density of the pipe plus water without the air.

The density is shown in Eq. (15).

$$
\rho=\frac{\rho_{\text {pipe }} \cdot A_{\text {pipe }}+\rho_{\text {water }} \cdot A_{\text {water }}}{A}
$$

where, $A_{\text {water }}$ is the cross-sectional area occupied by water; and $A_{\text {pipe }}$ is the cross-sectional area of the pipe.

In a two-phase water-air flow, the $A_{\text {water }}$ will directly depend on the amount of air present $H_{g}$, or void fraction. It can then be replaced by a more general expression where the area occupied by water, it is the volume fraction multiplied by the cross section area of the pipe, as shown in Eq. (16).

$$
A_{\text {water }}=A_{\text {pipe }} \cdot H_{L}=A_{\text {pipe }}\left(1-H_{g}\right)
$$

It can be concluded that the natural frequency of the cylinder has an indirect relation with the void fraction. Vibrations, induced by pressure fluctuations on the flow pattern, are thus capable of generating pressure fluctuations in the surrounding air, with enough power to be acquired by a microphone. The global vibrations of a long pipe segment, depending on its boundary conditions, will not be acoustically efficient. Nevertheless, the local vibrating modes of the pipe walls can be assessed and will be influenced by the nature of the flow in the interior of the pipe.

\section{Experimental Modeling}

The goal is to make the measurement of void fraction through the acoustic response of the system without being intrusive to the flow or pipe in real time, and an experimental was built in order to insert air flow in water and obtain the desired bi-phasic mixture. The control of the amount entering each stage will create the flow pattern. Indirectly, this control will impose a certain volume of air and a certain volume of water for a given test volume. Thus, it can obtain void fractions ranging from 0 to $100 \%$. The natural frequency of the system is related to the actual void fraction in the flow. The greater amount of water in the system, the larger the mass to be moved. Therefore, the periods of the natural vibration modes of the pipe wall would be higher, corresponding to lower frequencies. The variation of these natural frequencies of the system, as a response to variations of void fraction of the flow can 
be used as a metric. In a local method, this effect of the pulsating modes of pipe vibration will be captured by microphones.

\subsection{Flow Patterns and Calibration}

In order to implement the method, it is necessary to establish the relation between vibration frequency and void fraction [5]. In order to obtain that, an experimental set-up was built consisting of a tube closed at their ends, suspended by nylon threads as a free-free support. It is filled with water with volume proportions from 0 to $100 \%$, increasing in steps of $10 \%$. Therefore, a total of 11 spectra are available to be measured. Fig. 1 shows schematically the apparatus.

The procedure starts with the tube filled with a known volume of water. It is progressively emptied and the mass of the liquid is measured with a precision electronic scale. In each step, the pipe vibrations are excited with an impact hammer. The vibratory response of the system is assessed acoustically with microphones in the form of noise transfer functions. The LabVIEW ${ }^{\circledR}$ software system is used to read the data. Table 1 lists the sensors and actuator used.

Spectra are generated from 8,192 samples acquired with a rate of $25.6 \mathrm{kHz}$. Frequency resolution is, therefore, $3.125 \mathrm{~Hz}$.

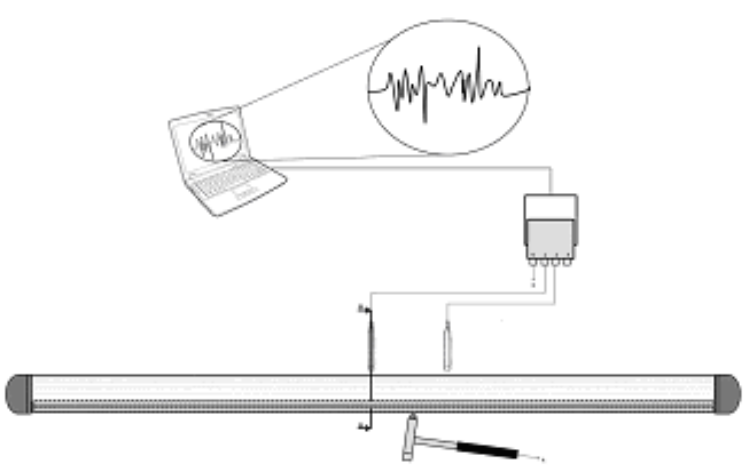

Fig. 1 Determining natural frequencies.

Table 1 Description of sensors and actuators used.

\begin{tabular}{lll}
\hline Sensor & Model & Sensibility \\
\hline Impact hammer & 086C03-PCB & $-5 /+5 \mathrm{Volts}$ \\
Microphone 01 & MPA416-BSWA & $46.8 \mathrm{mV} / \mathrm{Pa}$ \\
Microphone 02 & MPA416-BSWA & $45.7 \mathrm{mV} / \mathrm{Pa}$ \\
\hline
\end{tabular}

Fig. 2 exemplifies the frequency spectrum generated.

Fig. 3 shows in more detail, in a narrower frequency range, the pitch changes in the generated sound from the vibrating pipe.

From these observations, a calibration chart can be plotted. Seven different vibration modes can be found in the observed frequency range: (1) $125-150 \mathrm{~Hz}$; (2) 340-410 Hz; (3) 650-800 Hz; (4) 1,070-1,300 Hz; (5) 1,580-1,920 Hz; (6) 2,150-2,630 Hz; and (7) 3,650-4,350 Hz. In Fig. 4, one of the curves, relating natural frequency and void fraction, is shown with its respective uncertainties.

With these relations established, the technique was used in an experimental rig with water-air flow.

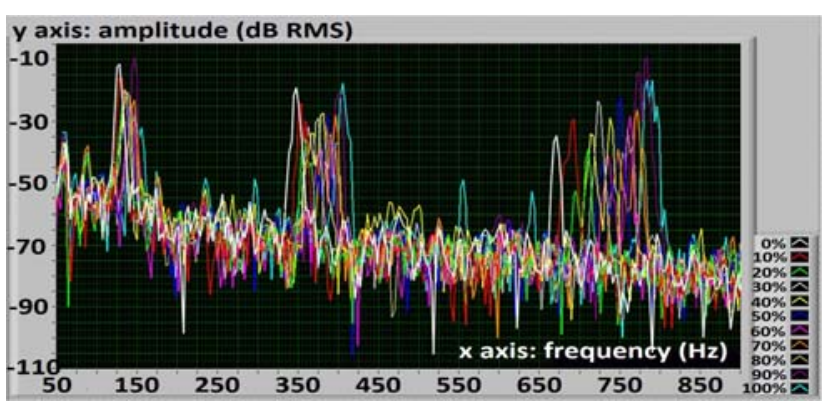

Fig. 2 Spectra showing the 11 measurements for each void fraction percentage.

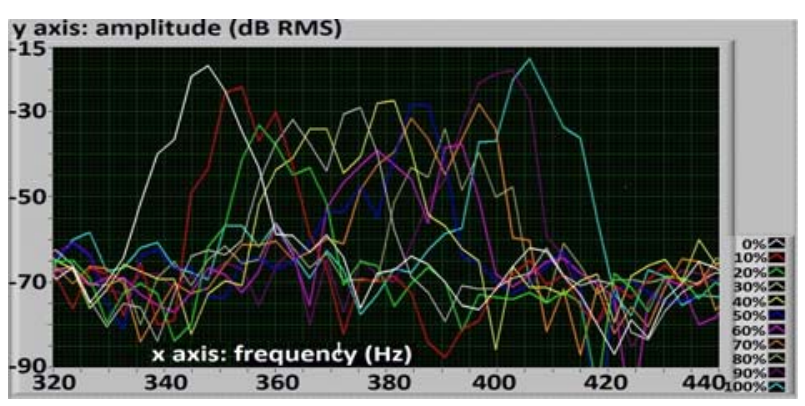

Fig. 3 The variation in only one frequency band.

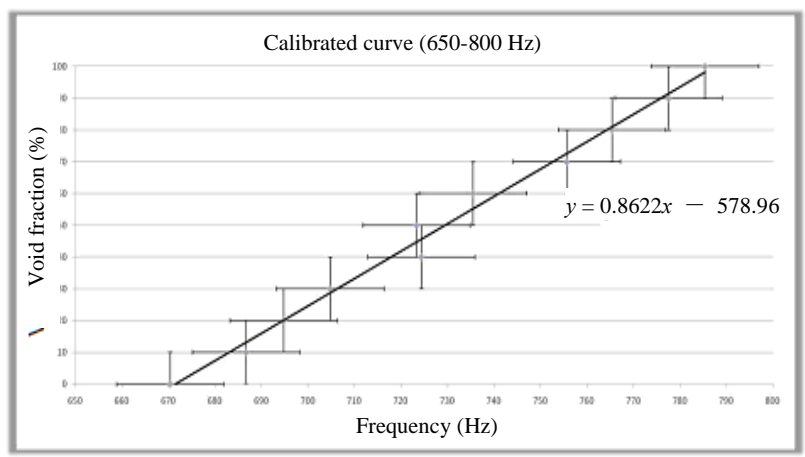

Fig. 4 Relation between natural frequency and void fraction for the vibrations in the range of $650 \mathrm{~Hz}$ to $800 \mathrm{~Hz}$. 


\subsection{Experimental Rig}

In order to make the measurements in flow conditions, a test cell is built in an experimental rig, and it is cyclically excited with an electromagnetic hammer. The rig is shown schematically in Fig. 5.

The outflow of a centrifugal pump is directed through a piping line with the measuring cell mounted on a steel pipe section. Behind this section, air coming from a compressed air line is fed into the flow. The amount of air introduced is controlled by a pressure regulator.

Vibration of the test cell is induced by repeatedly exciting it with the electromagnetic hammer in a $4 \mathrm{~Hz}$ rate. The measurements are done with the signal acquisition of $250 \mathrm{~ms}$ - the interval between each excitation [6]. Signal processing begins with data loading in the time domain, calculation of the power spectrum, and reading the peaks of the frequency bands, according to the pattern of curves previously obtained statically. The comparison of the curves calibrated for different vibration modes, leads to the possible value of the void fraction. The obtained values for the different bands and two different microphones, are then averaged to form a unique result for the void fraction. This unique result is presented by the software.

In order to reduce the effects of background noise, the test cell is isolated under a cylindrical shell, fitted on the outer diameter of the pipe. This shell also supports and locates the microphones and the excitation system. It can be mounted directly on the pipe without being necessary to disturb the flow.

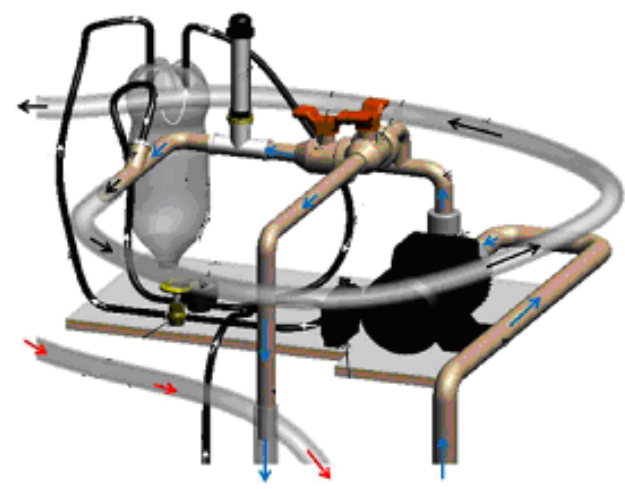

Fig. 5 The experimental rig of two-phase flow.
The instrumentation embedded in the cell consists of two microphones arranged radially, a mechanical mechanism of excitation and its electronic power drive. The microphones used have a frequency range from 20 $\mathrm{Hz}$ to 20,000 Hz. The excitation mechanism exerts an impact on the pipeline, so that the noise can be picked up by the microphones. Fig. 6 shows schematically the assembly, and the location and positioning of the measuring cell.

\subsection{Measurement Hardware and Analysis}

To process and analyze the data obtained from the experiment, a battery power excitation mechanism, a signal generator as the excitation control, a four channel acquisition module model NI 9233, a USB interface model NI 9162, and a notebook with LabVIEW software are used.

\section{Results}

The power spectra of two microphones placed in the measuring cell and a third microphone identical to the other two outside the cell measured simultaneously are presented. Fig. 7 shows the noise reduction of the casing, from $20 \mathrm{~dB}$ to $40 \mathrm{~dB}$ in the frequency range.

Initially, as a parameter verification, the extreme situations of 0 and $100 \%$ void fraction will be tested. These situations are similar to the test section completely filled with flowing water and flowing air, respectively. Figs. 8 and 9 present the results for different conditions of two phase flow.

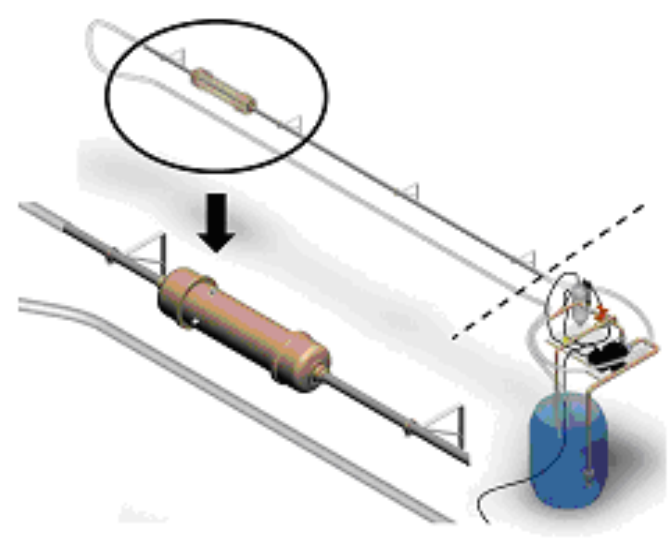

Fig. 6 The generalized view of the assembly. 


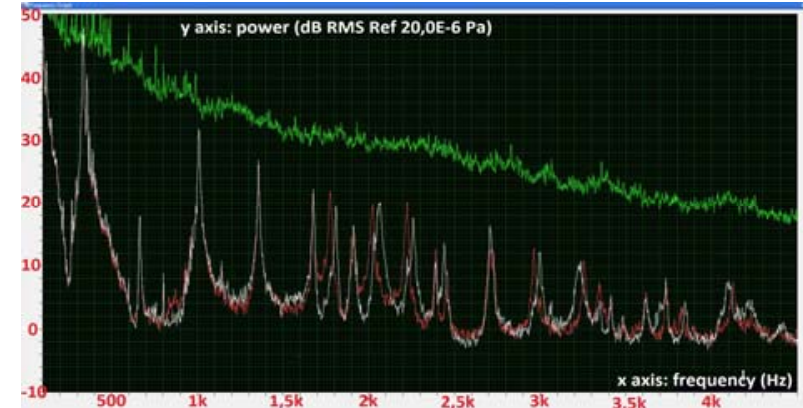

Fig. 7 Noise reduction (spectra in white and red are from microphones inside the measuring cell, green one for the outside microphone).

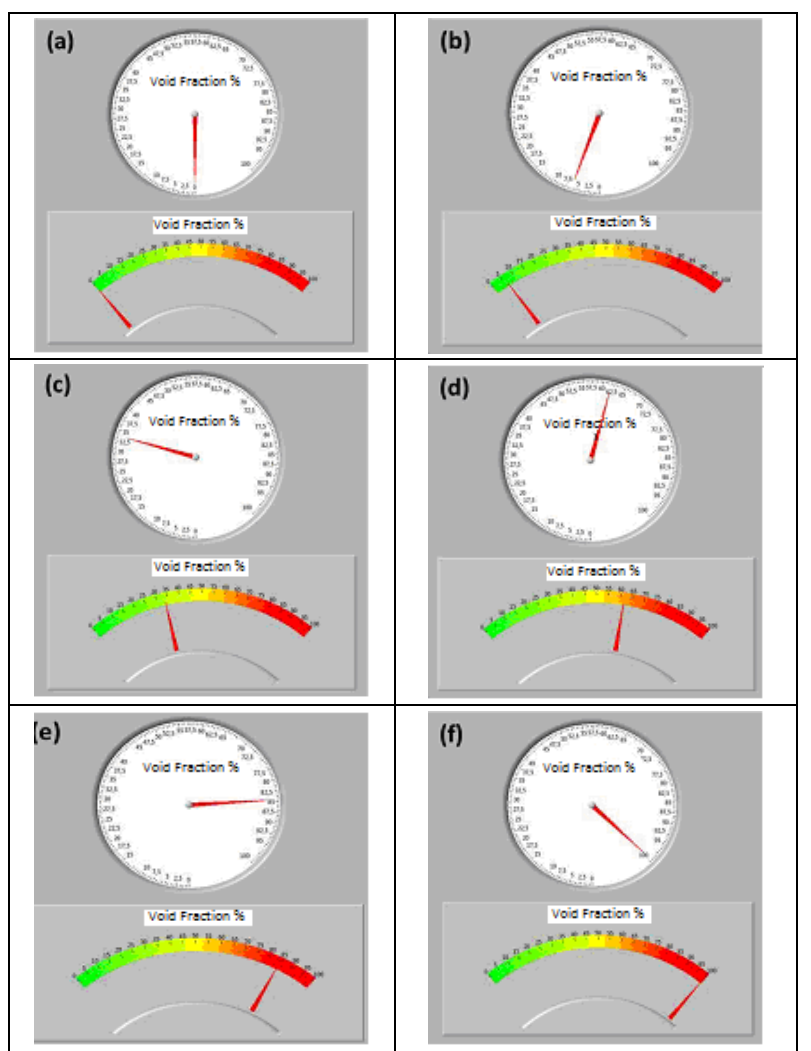

Fig. 8 Front panel indicators for results in real time: (a) 0\%; (b) $5 \%$; (c) $30 \%$; (d) $60 \%$; (e) $85 \%$ and (f) $100 \%$.

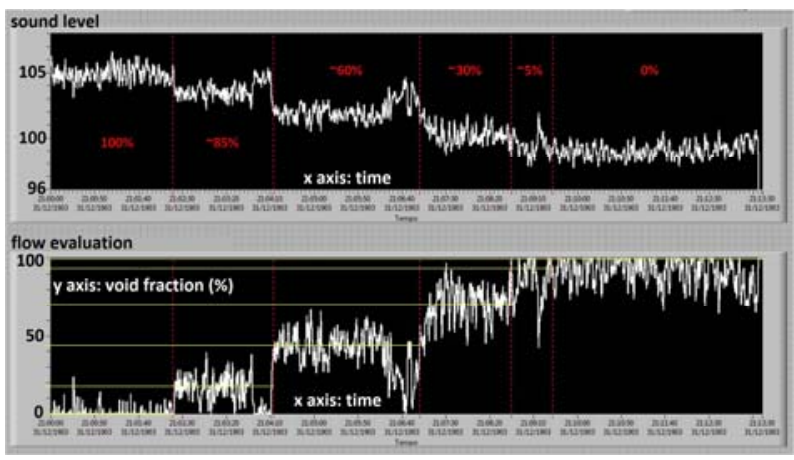

Fig. 9 Curve of the sound level and the evolution of the flow verification method.

\section{Conclusions}

The work presents a method to determine the void fraction in bi-phasic flows of the form of liquid and gas, based on the analysis of the acoustic response of the pipe wall. It is implemented and verified experimentally. It relates to other systems based on global vibration of the pipe. However, the local behavior of the pipe wall appears to be more sensitive to void fraction variations. The calibration curves were generated in a statical measurement. The criteria developed, combining different microphones and the response of different vibrating modes, showed good results. A dimensionless relation may be further developed in order to allow the easy application of the method to different pipe diameters. The construction of the experimental rig allowed the establishment of different floe patterns and void fractions. However, it may be further enhanced with flow instrumentation and a better control of the flow conditions. Currently, only indirect measurements, through pressure transducers are made. The method allows real-time measurement, which is not intrusive and low-cost, identifying the void fraction in two-phase flow in industrial pipes.

\section{Reference}

[1] Silva, R. D. 1985. "Void Fraction Measurement of Two-Phase Flow in Vertical Pipes Using Gamma Rays.” master thesis, Instituto de Pesquisas Energéticas e Nucleares.

[2] Dowling, A. P., and Williams, J. E. 1983. Sound and Sources of Sound. Chichester: Ellis Horwood Limited.

[3] Filho, P. H. A. W. 2010. "Method for Determination of Volumetric Fractions of Two-Phase Flow Based on Analysis of Frequency Response Functions of the Pipe.” master thesis, Universidade Federal Fluminense.

[4] Gama, A. L., Moreira, R. M., and Oliveira, F. N. 2006. "Procedures for Evaluation and Inspection Pipes Featuring Excessive Vibration." In Proceedings of the First Congress Reliability, Inspection and MaintenancePETROBRAS, 104-29.

[5] Hall, D. E. 1987. Basic Acoustic. New York: Harper \& How.

[6] Holman, J. P. 1994. Experimental Methods for Engineers. 6th edition. Singapore: McGraw-Hill Inc. 
[7] Riverin, J. L., and Pettigrew, M. J. 2006. "Vibration Excitation Forces due to Two-Phase Flow in Piping Elements.” Journal of Pressure Vessel Technology 129 (March): 7-13.

[8] Mandhane, J. M., Gregory, G. A., and Aziz, K. 1974. A Flow Pattern Map for Gas-liquid Flow in Horizontal Pipes. Elsevier 74: 90006-8.

[9] Evans, R. P., Blotter, J. D., and Stephens, A. G. 2008. "Flow Rate Measurements Using Flow-Induced Pipe Vibration.” Journal of Fluids Engineering 162: 280-85.
[10] Beck, M. S., Green, R. G., and Thorn, R. 1987. "Non-intrusive Measurement of Solids Mass Flow in Pneumatic Conveying.” Journal Physics E: Science Instrument 20 (7) 835-42.

[11] Chan, K. T., and Zhang, J. Z. 1995. "Free Vibration of a Cantilever Tube Partially Filled with Liquid.” Journal of Sound and Vibration 182 (April): 185-90.

[12] Alkow, K. J., and Rezkallah, K. S. 1996. "Void Fraction Measurements in Gas-liquid Flows Using Capacitance Sensors.” Measurement Science and Technology 23: 815-29. 